\title{
Real-Time Performance Assessment of Operating Photovoltaic (PV) Systems
}

\author{
Abdullah M. ALzahrani*, Mohamed A. Zohdy\# \\ Department of Electrical and Computer Engineering, Oakland University, Rochester, MI, USA \\ Email: alzahrani2@oakland.edu, zohdyma@oakland.edu
}

How to cite this paper: ALzahrani, A.M. and Zohdy, M.A. (2020) Real-Time Performance Assessment of Operating Photovoltaic (PV) Systems. Energy and Power Engineering, 12, 339-347.

https://doi.org/10.4236/epe.2020.126020

Received: March 3, 2020

Accepted: June 16, 2020

Published: June 19, 2020

Copyright $\odot 2020$ by author(s) and Scientific Research Publishing Inc. This work is licensed under the Creative Commons Attribution International License (CC BY 4.0).

http://creativecommons.org/licenses/by/4.0/

\begin{abstract}
A technology called solar energy is a very promising technique, and is considered as the cleanest and the most abundant renewable resource that is naturally available every day. In this paper, a MATLAB environment has been developed to calculate real-time power incidence on a PV system. It takes into account the time, location, PV tilt, and azimuth angles, and weather conditions to estimate incident power. In this paper, one case study is considered at New York State location. It has been applied to a newly installed $8 \mathrm{~kW}$ residential system located in Inwood. The solar panels are made up of silicon HIT (Heterojunction with Intrinsic Thin Layer) cells by Panasonic and solar cell rated at $19 \%$. The result shows that the system is performing at its rated efficiency. The calculations involve the determination of direct, diffused and reflected radiation on the panels taking into account the time of the day, location, PV area, and orientations and weather conditions. The cloudiness index may be estimated based on the weather data and included in the calculations. After performing the irradiance calculations, the output power is estimated based on the rated efficiency at its temperature and compared with the generated output power. The real-time assessment of a PV system performance, during the operational time monitors the health of the PV system. The data obtained by this calculator may accompany production data provided to the consumer by the utility company.
\end{abstract}

\section{Keywords}

PV System, Irradiance Calculation, PV Performance Method

\section{Introduction}

These days' traditional resources, such as fossil fuels and natural gas, are known to be restricted resources in the main time and may produce air pollution emis${ }^{\star}$ Ph.D. student.

"Professor. 
sions which is of great concern to the environment and problems such as global warming. Conversely, utilizing clean energy sources, such as solar energy has become a major player in the solution for the energy crisis in the near future.

Consequently, this clean energy technology has widely spread in the field of power systems and production. It has been significantly modified and improved throughout diverse stages of progress [1]. These stages of modification and improvement experienced by this field are the outcomes of several factors which have both harmed and benefited the development of clean energy technology. Today, the world economies are more drastically distinct than they were previously. The clean power energies have been developed at a stage much faster than some other analysts have predicted, because these technologies had been supported and developed by some researches and national governments [2]. Thus, the international communities such as the United States Environmental Protection Agency (EPA) have set extra stress and work on developing clean energy in terms of reducing the need of using fossil fuels and other energy sources that can negatively affect the environment.

Therefore, solar energy systems are gaining popularity among these clean energy sources which are highly recommended for the environment. To raise its popularity, it is critical to improving the efficiency of solar energy capabilities to convert light-to-electricity energy. Based on that, the efficiency of the solar system has significantly been improved utilizing new technologies [3]. These improvements have forced the need for the photovoltaic (PV) system to be a significantly important system in the generation of electrical power worldwide.

Accordingly, the main function of all these PV systems is to convert solar radiation into electricity [4]. Wherefore, there are several types of photovoltaic systems in the market that have been designed in terms of providing energy to meet the needs of electricity with very high efficiency at the lowest cost. Based on that, there are two ways to ensure a better efficiency of the PV system. One is to study the behavior of the PV system while increasing the temperature, shadow effects on solar PV arrays, spectral mismatch and so on in the outdoor environment [5].

Moreover, observing the performance of photovoltaic systems have become a fundamental mission to have a robust and reliable system. The observation of the performance can offer beneficial information about the photovoltaic's operation and can designate what is required to be done to ameliorate the performance of the system if the dataset is conveyed correctly, in the opposite, the endeavors are wasted. This observation can also give a piece of good information on relevance of the operation to a third party in terms of notifying them about their PV arrays in case of unexpected events. For instance, if the real power generated from a solar PV array falls far below the designed level, which at times, can lead to a complete loss in the efficiency. Moreover, if shading happens it can cause a long term performance loss, hence the shortfall in the expected level of annual power production [6].

In this paper, an assessment of the performance of the PV system is used in 
terms of involving a third party to be aware of the performance of the PV system that is installed on their property. The system output can be sensed every minute, or every hour and the results stored. Even with hourly or minutely sensed output, a huge amount of dataset is generated. To be useful, the raw dataset must be checked for integrity and then summarized. Data needs to be collected for at least twelve months to reflect seasonal variations, even though monthly averages are a convenient interval for summarizing energy production, consumption, losses and efficiency [7].

\section{The Sun Position and Solar Angles}

There are three main factors to locate the sun's position in the sky [8]. These factors can be described as the solar altitude $\alpha$, the solar azimuth $\Psi$ and the solar angle of incidence $\theta$. These angles are specifically defined based on given geographical location, its latitude $L$, longitude $\phi$, at a given time of day and year. On the other hand, there are other angles related to solar panels that have to be taken into consideration, namely, the slope surface tilt angle $\beta$ and surface azimuth angle $\gamma$ [8]. In the following, the description and formulation for each angle is given. Yet, before giving the formulation of solar altitude and azimuth angles, the solar declination $\delta$ and hour angle $\omega$ have to be addressed in all other solar angle formulations.

\subsection{Declination Angle, $\delta$}

Declination is the angle that locates between the sun's direction and the orbit plane of the earth around the sun. It changes generally as a result of the rotation of earth around an axis. The maximum value is $23.45^{\circ}$ at midsummer in the northern hemisphere and the minimum value is $23.45^{\circ}$ at northern midwinter [9]. It can be expressed as follows

$$
\delta=\varepsilon \sin \left\{\frac{(n+284) \times 360}{365}\right\},
$$

where, $\varepsilon=23.45^{\circ}$ is constant value, and $n$ represents the day of the year.

\subsection{Solar Altitude Angle, $\alpha$}

The solar altitude angle, $\alpha$, is the solar angle that is located between the horizontal and the solar beam, that is, the complement of the zenith angle. It can be given as follow

$$
\alpha=\sin ^{-1}\{\sin (L) \sin (\delta)+\cos (L) \cos (\delta) \cos (\omega)\},
$$

where, the hour angle is $\omega=15$ (localTime-12). Therefore, during morning time the solar altitude angle rises until achieving the peak at noon which matches the sun's crucial elevation in the sky with regarding to an observation point on the earth [9].

\subsection{Solar Azimuth, $\Psi$}

The solar azimuth angle, $\Psi$, is the sun's deviation of the projection from the 
north hemisphere is zero for a surface facing due south, $180^{\circ}$ due north, $0^{\circ}$ to $180^{\circ}$ for a surface facing westwards and, $0^{\circ}$ to $180^{\circ}$ eastward [9].

$$
\begin{gathered}
\left\{\begin{array}{c}
\Psi=180-\cos ^{-1}(\cos (\Psi)), \text { if } \sin (\Psi)<0 \\
\Psi=180+\cos ^{-1}(\cos (\Psi)), \text { if } \sin (\Psi)>0
\end{array}\right. \\
\sin \Psi=\frac{\cos (\delta) \sin (\omega)}{\cos (\alpha)}
\end{gathered}
$$

\subsection{Solar Incidence Angle, $\theta$}

The angle incidence, $\theta$, is the angle between the beam radiation on the surface and a vector orthogonal to the surface of a solar panel on the ground, it can be expressed as

$$
\begin{aligned}
\cos (\theta)= & \sin (\beta) \sin (L) \cos (\beta)-\sin (\delta) \cos (L) \sin (\beta) \cos (\gamma) \\
& +\cos (\delta) \cos (L) \cos (\beta) \cos (\omega)+\cos (\delta) \sin (L) \cos (\gamma) \cos (\omega) \\
& +\cos (\delta) \sin (\beta) \sin (\gamma) \sin (\omega)
\end{aligned}
$$

\subsection{Solar Radiation on the Earth's Surface}

In this experimental study, the three-component model of the solar irradiance is considered to compute the total solar irradiance on an inclined surface that can be expressed as $S_{G}^{T}$, the superscripts $T$ indicates tilted surfaces, arriving at an inclined PV-module

$$
S_{G}^{T}=S_{B}^{H} \times\left(\frac{\cos \theta}{\cos z}+\frac{\rho(1-\cos \beta)}{2}\right)+S_{D}^{H} \times\left(\frac{(1+\rho)+(\cos \beta-\rho \cos \beta)}{2}\right)
$$

where, $S_{B}^{H}$ represents the direct beam irradiance measured at the horizontal surface that reaches the PV-module without being reflected or scattered, $S_{D}^{H}$ represents the diffused solar irradiance on horizontal surfaces which is scattered by the clouds and the reflection coefficient $\rho$ is the reflected solar irradiance by the ground, see Figure 1 .

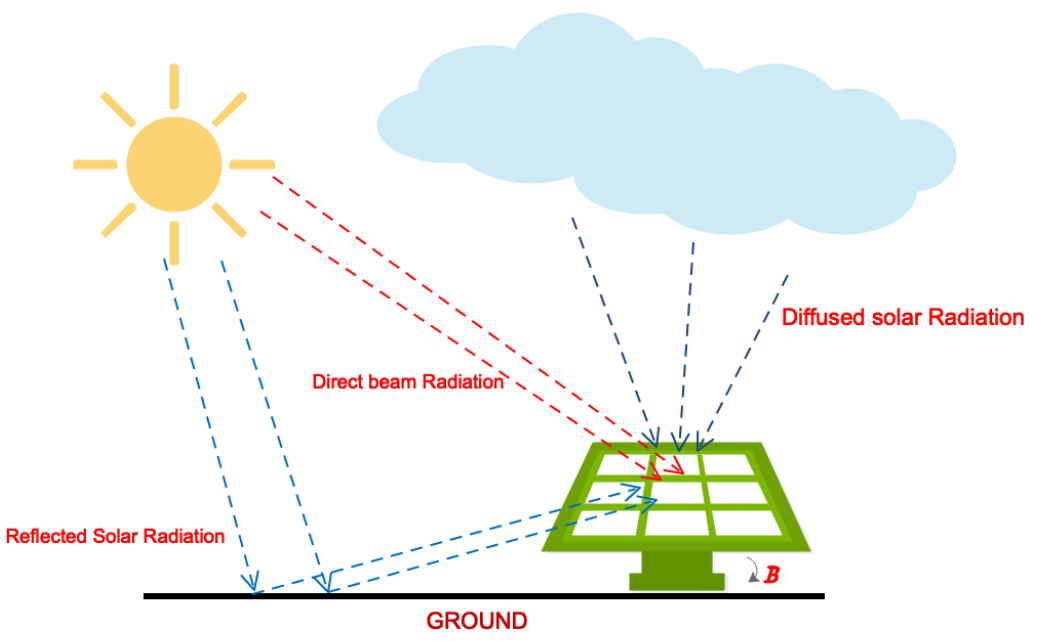

Figure 1. Definition of various solar irradiance on the PV model. 
Therefore, as mentioned solar irradiance components can be defined as follows

- The direct beam irradiance on a horizontal surface can be defined by

$$
S_{B}^{H}=I_{0} \times\left\{1+0.033 \cos \left(2 \pi \frac{n-4}{365}\right)\right\} \times \mathrm{e}^{-0.8662 T_{L}(m) D_{R}(m)} \cos (z)
$$

where, $I_{0}$ represents the solar constant, known as $1360 \mathrm{~W} / \mathrm{m}^{2}$, the Linke turbidity factor is represented by $T_{L}$, the Rayleigh scattering optical depth is represented by $D_{R}(m)$, and air mass is represented by $m$ [10]. Thus, the air mass $m$ equation is formulated by Kasten Young (1989) in [10] as

$$
m=\frac{1}{\cos z+0.50572 \times(96.07995-z)^{-1.6364}}
$$

Moreover, the Linke turbidity factor, $T_{L}$ is described as the number of Rayleigh atmospheres necessary to exemplify the actual optical thickness of $D(m)$ as

$$
T_{L}=\frac{D(m)}{D_{R}(m)},
$$

where the empirical equation for $D_{R}(m)$ is given by [10] as follows

$$
D_{R}(m)=\frac{1}{6.6296+1.7513 m-0.1202 m^{2}+0.0065 m^{3}-0.00013 m^{4}}
$$

- The diffused irradiance on a horizontal surface is defined by [11].

Therefore, a useful direction that can be used to define the extent of cloudiness of the sky is through some mathematical relationships among the available solar fluxes like global irradiance, diffuse irradiance and extraterrestrial irradiance. Two parameters inter-relating these fluxes are defined as the diffuse fraction of horizontal radiation, $K_{D}$ and the clearness index, $K_{C}$. These two parameters thus can be expressed as

$$
\begin{gathered}
K_{D}=\frac{S_{D}^{H}}{S_{G}^{H}} \\
K_{C}=\frac{S_{G}^{H}}{\text { Extraterrestrial horizontal irradiance }}
\end{gathered}
$$

thus, in this work [11], the study determined that solar radiation characteristics can be utilized to defined the following model of diffused fraction related to the clearness index as

$$
K_{D} \approx \begin{cases}0.98, & \text { if } K_{C} \leq 1 \\ 0.910+1.15 K_{C}-4.936 K_{C}^{2}+2.848 K_{C}^{3}, & \text { if } 0.1 \leq K_{C} \leq 0.7\end{cases}
$$

\section{Experimental Study}

In this study, a solar radiation program is done using the MATLAB environment. The program designed to accept the values of the geographical location such as latitude $L$, longitude $\phi$, at any given time of day and year and the panel tilt an- 
gle along with the azimuth angle as inputs. This program is designed to define the time of the sunset and sunrise for any solar panel. Thus, it can be also used to calculate daily irradiance on the solar panels. In this study, a Panasonic HIT solar cell is considered to evaluate the system performance during a specific day of operation at the location of Inwood, New York. The parameters used in irradiance calculations are given in Table 1.

Table 1. Parameters of irradiance that is used in calculations, at Inwood, New York.

\begin{tabular}{cc}
\hline Type & Parameters \\
\hline Latitude & $44.16 \mathrm{~N}$ \\
Longitude & $77.16 \mathrm{~W}$ \\
Day & $5^{\text {th }}$ \\
Month & 3 \\
Year & 2020 \\
Module Tilt Angle, $\beta$ & 27 \\
Modules ${ }_{1}$ Azimuth Angle, $\Psi_{1}$ & $61 \mathrm{E}$ \\
Modules ${ }_{2}$ Azimuth Angle, $\Psi_{2}$ & $241 \mathrm{SE}$ \\
Solar Constant & $1360 \mathrm{Wm}^{-2}$ \\
Ground Reflectivity, $\rho$ & 0.2 \\
Linke Turbidity, $T_{L}$ & 1 \\
Clearness Index, $K_{C}$ & 0.5 \\
Number of Modules ${ }_{1}$ & 16 \\
Number of Modules ${ }_{2}$ & 9 \\
Module Area & $1.461 \mathrm{~m}^{2}$ \\
\hline
\end{tabular}

\section{Experimental Results}

In this study, the performance of a PV system depends mainly on solar irradiance and temperature. Evaluating the system efficiency is significantly important in daily operations to provide sufficient energy to cover the need for electricity. On the other hand, there are some other factors that can effect the system performance such as dust, dirt, and shadows. These factors can be accumulated on the PV system HIT module surface, blocking some of the sunlight and reducing output [12].

Although typical dirt and dust is cleaned off during every rainy season, it is more realistic to estimate system outputs by taking into account the reduction due to dust build-up in the dry season [12]. As an example of that, if we have a system of a PV system HIT panels that is designed to operate at $2000 \mathrm{~kW}$, with some accumulated dirt/dust it may operate on average at about $1958 \mathrm{~kW}$ [12]. Therefore, regarding the energy conversion efficiency, it was evaluated according to the following relation

$$
\mu=\frac{P_{\text {production }}}{A \times I_{G}^{H}}
$$


where, $A$ represents the area surface on the panel, $P_{\text {production }}$ represents the electrical production and $I_{G}^{H}$ is the incoming solar irradiation. The temporal evolution of this solar system electrical efficiency is described in Figure 2.

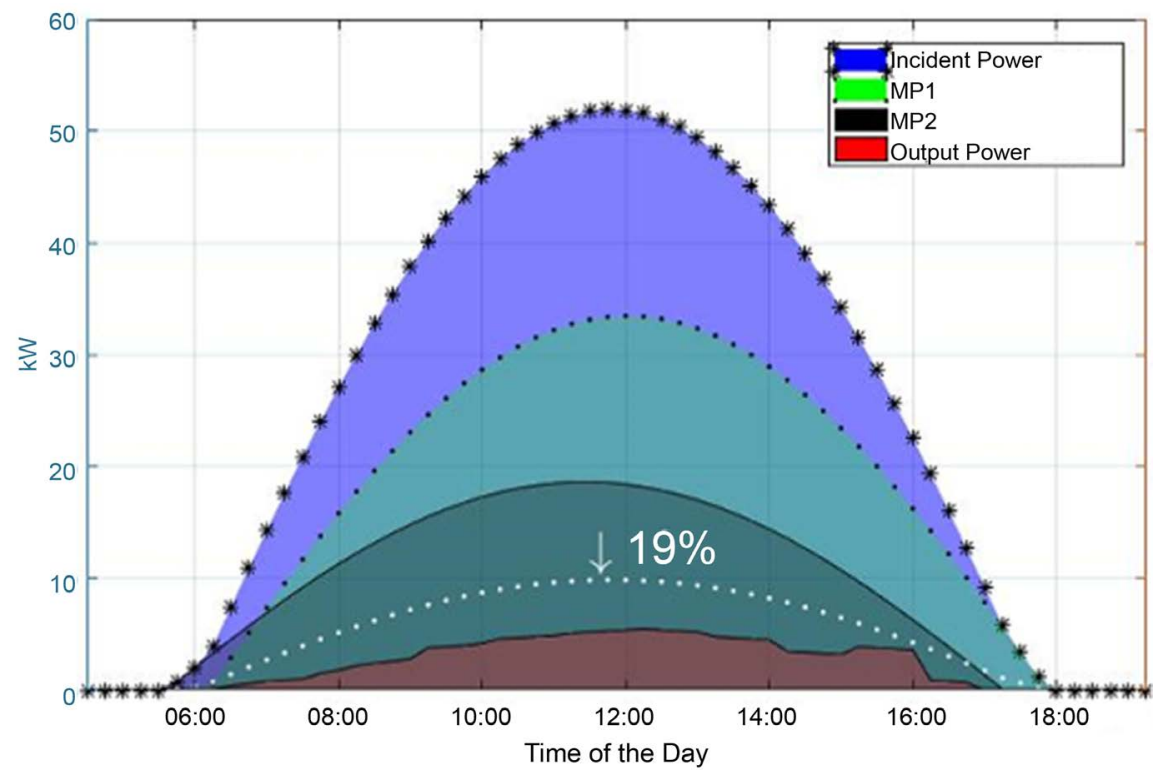

Figure 2. System performance.

It can be seen that the electrical efficiency is decreasing up to noon due to the negative effect of the solar cells temperature during this period of the day and it is increasing for the rest of the day. It is also noticed that the PV panel electrical efficiency is higher for white soil due to its higher electrical production. As a result of the given plot, the blue graph represents the total power of both models from sunrise at around 6:15 a.m. up to sunset at around 7:15 p.m., while the red graph is the power production at the homeowner during the day. By using the Equation (14), the system efficiency can be obtained based on the given parameters. Thus, the system efficiency is described by the white dotted line and shows that the system efficiency is $19 \%$. Therefore, the homeowners have daily access to track the system performance and they can report the system's insulators about their concerns based on the data reading. Thus, this program has the ability to provide and evaluate the system performance during each slot of operation.

\section{Conclusion}

In this paper, an experimental study of specific PV panels has been designed using the MATLAB environment to perform a daily evaluation on the efficiency and performance of the model. It has been done by using equations that have been formulated by extensive study of previous researches and extending the model to the present problem that is identified. The measurement of the PV system performance ensures, as an assessment of the health of the system, verification of a performance model to then be applied to another system. A per- 
formance assessment is most valuable when it is completed, yet currently, no standard exists to guide this process. The results of this research are very promising, with a considerable enhancement of the efficiency relative to the PV model. This model provides some advantages in order to evaluate the performance of the PV system during the operational time and to assess the health of the PV system. Moreover, this program can be also developed in solar tracking system to set or determine a good position for the PV panels where it can absorb more sunlight to provide enough energy to support the loads. Thus, this technique will help reduce the dependency of traditional sources which could be depleted in the near future by enhancing the use of solar energy, especially the PV model.

\section{Conflicts of Interest}

The authors declare no conflicts of interest regarding the publication of this paper.

\section{References}

[1] Dincer, I. and Acar, C. (2018) Potential Energy Solutions for Better Sustainability. In: Dincer, I., Colpan, C.O. and Kizilkan, O., Eds., Exergetic, Energetic and Environmental Dimensions, Academic Press, London, 3-37. https://doi.org/10.1016/b978-0-12-813734-5.00001-9

[2] Nidumolu, R., Prahalad, C.K. and Rangaswami, M.R. (2009) Why Sustainability Is Now the Key Driver of Innovation. Harvard Business Review, 87, 56-64. https://doi.org/10.1109/emr.2015.7123233

[3] Green, M.A., Emery, K., Hishikawa, Y., Warta, W. and Dunlop, E.D. (2012) Solar Cell Efficiency Tables (Version 40). Progress in Photovoltaics. Research and Applications, 20, 606-614. https://doi.org/10.1002/pip.2267

[4] Nishioka, K., Shimakage, T., Yamane, H., Kudo, M. and Ueda, Y. (2010) Evaluation of output Performance of Various Photovoltaic Systems in the Hokuto Mega-Solar Project. 32nd International Telecommunications Energy Conference (INTELEC), Orlando, FL, 6-10 June 2010, 1-7. https://doi.org/10.1109/intlec.2010.5525661

[5] Kim, Y.-B. and Song, B.-S. (2018) A Study on Module-based Power Compensation Technology for Minimizing Solar Power Loss due to Shaded Area. The Journal of the Korea Institute of Electronic Communication Sciences, 13, 539-546.

[6] Alsadi, S.Y. and Nassar, Y.F. (2019) A General Expression for the Shadow Geometry for Fixed Mode Horizontal, Step-Like Structure and Inclined Solar Fields. Solar Energy, 181, 53-69. https://doi.org/10.1016/j.solener.2019.01.090

[7] van Sark, W., et al. (2017) PV System Monitoring and Characterization. In: Reinders, A., Verlinden, P., van Sark, W. and Freundlich A., Eds., Photovoltaic Solar Energy, John Wiley \& Sons Ltd., Chichester, 553. https://doi.org/10.1002/9781118927496.ch49

[8] Scharmer, K. and Greif, J. (2000) The European Solar Radiation Atlas Vol.1: Fundamentals and Maps. Les Presses del Ecole des Mines, Paris, 23-42.

[9] Haberlin, H. (2012) Photovoltaics System Design and Practice. John Wiley \& Sons, Chichester.

[10] Kasten, F. and Young, A.T. (1989) Revised Optical Air Mass Tables and Approximation Formula. Applied Optics, 28, 4735-4738. 
[11] Fan, J., et al. (2019) Evaluation and Development of Empirical Models for Estimating Daily and Monthly Mean Daily Diffuse Horizontal Solar Radiation for Different Climatic Regions of China. Renewable and Sustainable Energy Reviews, 105, 168-186. https://doi.org/10.1016/j.rser.2019.01.040

[12] Alnasser, T.M.A., et al. (2020) Impact of Dust Ingredient on Photovoltaic Performance: An Experimental Study. Solar Energy, 195, 651-659.

https://doi.org/10.1016/j.solener.2019.12.008 